\title{
Dermatopontin as a potential pathogenic factor in endometrial cancer
}

\author{
HAIYUN HUANG ${ }^{1 *}$, ZHIXIANG HAO ${ }^{2 *}$, LINGYAN LONG ${ }^{1}$, ZEYUAN YIN $^{1}$, \\ CHENYU WU $^{1}$, XUEYAN ZHOU ${ }^{2}$ and BEI ZHANG ${ }^{3}$ \\ ${ }^{1}$ Department of Obstetrics and Gynaecology, Xuzhou Central Hospital, Affiliated Xuzhou Clinical College of \\ Xuzhou Medical University; ${ }^{2}$ Jiangsu Key Laboratory of New Drug Research and Clinical Pharmacy, \\ Xuzhou Medical University; ${ }^{3}$ Department of Obstetrics and Gynaecology, Xuzhou Central Hospital, \\ Affiliated Xuzhou Clinical College of Xuzhou Medical University, Xuzhou, Jiangsu 221004, P.R. China
}

Received November 17, 2020; Accepted February 23, 2021

DOI: $10.3892 / 01.2021 .12669$

\begin{abstract}
The present study aimed to determine the differential expression profiles of proteins in endometrial carcinoma and to screen the proteins associated with the occurrence and development of endometrial cancer (EC). In total, 15 samples of human EC and paracancerous tissues were selected for proteomic analysis using a label-free quantification method based on liquid chromatography-tandem mass spectrometry. The differential proteins were analysed using bioinformatics and verified using reverse transcription-quantitative PCR (RT-qPCR) and western blotting. Finally, the expression of differential proteins in 75 endometrial carcinoma samples and 30 normal endometrial tissue samples were detected using immunohistochemical staining, and the associations between differential protein expression and clinicopathological features were analysed. In total, 579 up-regulated proteins and 346 down-regulated proteins were identified between the two groups and seven proteins with the most significant differences were selected; these proteins included interferon-induced protein with tetratricopeptide repeats 3, poly(ADP-ribose) polymerase family member 9 , solute carrier family 34 member 2 , cytochrome b5 reductase 1 , protein tyrosine phosphatase non-receptor type 1, dermatopontin (DPT) and secretory leukocyte peptidase inhibitor. RT-qPCR and western blotting showed that DPT expression was down-regulated $(\mathrm{P}<0.001)$, which was consistent with the mass spectrometry results. The
\end{abstract}

Correspondence to: Dr Bei Zhang, Department of Obstetrics and Gynaecology, Xuzhou Central Hospital, Affiliated Xuzhou Clinical College of Xuzhou Medical University, 199 South Jiefang Road, Xuzhou, Jiangsu 221004, P.R. China

E-mail: author@mail.com

*Contributed equally

Key words: endometrial cancer, dermatopontin, proteomics, liquid chromatography-tandem mass spectrometry, biomarker, reverse transcription quantitative PCR immunohistochemical staining results showed that the positive expression of DPT in EC and normal endometrial tissues was statistically significant $(\mathrm{P}<0.001)$. The positive expression of DPT was significantly decreased in poorly differentiated, late stage, lymph node metastasis and myometrial invasion depth $\geq 1 / 2$ samples $(\mathrm{P}<0.05)$. DPT expression was significantly lower in EC, which might play role in the pathogenesis of EC.

\section{Introduction}

Endometrial cancer (EC) is a group of endometrial malignant tumours that often occur in perimenopausal and postmenopausal women. With $>300,000$ newly diagnosed tumours worldwide every year, EC is the third most common gynaecological malignant tumour (1). In the last 10 years, the incidence of EC has been increasing in China, ranking second only to cervical cancer among female reproductive system malignant tumours; however, the molecular mechanism of its pathogenesis has not yet been elucidated (2).

Currently, EC lacks specific markers for diagnosis and treatment and clinical diagnosis depends on endometrial biopsy, which is obtained using invasive methods. When EC is in the early stages of the disease the cure rate is as high as $83 \%$ but the survival rate decreases sharply to $<20 \%$ after late diagnosis (2). Only $\sim 17 \%$ of patients with distant metastasis can be cured $(3,4)$. Previous data have demonstrated a significant increase in the proportion of patients with advanced EC where survival rate has been minimal despite improved treatments (5).

Proteomics have been extensively studied in various types of cancer and potential biomarkers of EC have been studied, including serum amyloid $A$ and $\alpha-1-\beta$ glycoprotein $(1,6,7)$. In the present study, proteomics technology was used to detect the differential protein expression profiles in human EC and paracancerous tissues.

DPT is a tyrosine-rich extracellular matrix protein originally isolated from bovine dermal extracts that increases the formation of collagen fibres (8). DPT has a variety of biological functions in pathophysiological processes, such as regulating the interaction between decorin and transforming growth factor- $\beta$ (TGF- $\beta$ ) and increasing the biological activity of 
TGF- $\beta$ (9). In addition, DPT has an R-G-A-T sequence that can interact with integrins and is similar to the R-G-D sequence that binds integrins (10). DPT combines with the extracellular matrix receptor integrin $\alpha 3 \beta 1$ and promotes the formation of abnormal blood vessels in the tumour microenvironment by regulating TGF- $\beta$ and integrin $\alpha 3 \beta 1$ (11).

The present study purposed to detect the differential proteins in EC using a label-free quantification (LFQ) method based on liquid chromatography-tandem mass spectrometry (LC-MS/MS) and to investigate the proteins associated with the development of EC. It was first elucidated the expression of DPT in EC by RT-qPCR, western blotting and IHC. Furthermore, the current study provided evidence demonstrating that the expression level of DPT might be intimately related to the pathogenesis of EC.

\section{Materials and methods}

Tissue specimens. Tissue samples from EC resection operations were collected from the Department of Obstetrics and Gynaecology of Xuzhou Central Hospital (Xuzhou, China) between January 2017 to June 2020. Postoperative pathology performed by independent pathologists confirmed endometrial carcinoma in 15 cases of cancerous tissue and paracancerous tissue samples. A total of $75 \mathrm{EC}$ tissue wax blocks were collected from female patients with a mean age of 51.7 years (range, 42-76 years), and staged according to the International Federation of Gynaecology and Obstetrics [International Federation of Gynaecology and Obstetrics (FIGO) 2009] (12). Thirty normal endometrial tissue wax blocks were taken from female patients with an average age of 50.9 years (range, $46-58$ years) who underwent total hysterectomy due to uterine fibroids or adenomyomas during the same time period. The postoperative pathological identification of these tissues was performed by independent pathologists and showed a normal proliferative phase of the endometrium.

Tissue samples were collected within $30 \mathrm{~min}$ after the removal of the uterine appendages. Paracancerous tissues were defined as the tissue within $3-\mathrm{cm}$ outside of the $1-\mathrm{cm}$ edge of the cancer tissue. Paracancerous tissues were collected first, followed by cancer tissues. Then, the samples were cut into multiple tissue blocks with a diameter of $\sim 0.5-\mathrm{cm}$ and stored in a $-80^{\circ} \mathrm{C}$ freezer. All the cancer tissue samples were pathologically diagnosed as endometrioid carcinoma and all the paracancerous tissue samples were pathologically diagnosed as non-tumour tissue invasion in the pathology department of Xuzhou Central Hospital. All subjects met the following criteria: i) Pathological diagnosis of endometrioid carcinoma, ii) complete clinical characteristics, iii) no preoperative chemoradiotherapy or endocrine therapy and iv) no history of chronic disease or other malignant tumour. This study was approved by The Ethics Committee of Xuzhou Central Hospital (Xuzhou, China) and all patients provided informed written consent.

Proteomics analysis. A total of three cancer and three paracancerous tissue samples were selected from the 15 tissues collected from the Department of Obstetrics and Gynaecology. After weighing and lysis using $8 \mathrm{M}$ urea (Sigma-Aldrich; Merck KGaA) and $1 \%$ protease inhibitor cocktail (Thermo
Fisher Scientific, Inc.)], the supernatant was collected to determine the protein concentration of the samples. Equal amounts of proteins $(\sim 50 \mu \mathrm{g})$ from samples were reduced with $10 \mathrm{mM}$ dithiothreitol (Thermo Fisher Scientific, Inc.) and alkylated with $50 \mathrm{mM}$ iodoacetamide (Bio-Rad Laboratories, Inc.). Solution digestion was then performed with sequencing grade modified trypsin at $37^{\circ} \mathrm{C}$ overnight. The peptides were acidified with a final concentration of $0.5-1 \%$ trifluoroacetic acid (Thermo Fisher Scientific, Inc.). Sodium deoxycholate (Sigma-Aldrich; Merck KGaA) was removed using high-speed centrifugation $\left(14,000 \mathrm{x} \mathrm{g}\right.$ at $4^{\circ} \mathrm{C}$ for $\left.20 \mathrm{~min}\right)$. Tryptic peptides were desalted and centrifuged in a SpeedVac to dry. Then, tryptic peptides were redissolved in $0.1 \%$ formic acid (Thermo Fisher Scientific, Inc.).

After the enzymatic hydrolysis and pre-treatment of the samples, the LFQ method based on LC-MS/MS technology was used for peptide separation and mass spectrometry analysis of each sample by higher-energy collisional dissociation (HCD) (ionization mode, positive). LC-MS/MS was conducted to obtain primary mass spectrometry of all ions and the first 20 peptides with the signal strength of the primary spectrum peak were identified using secondary MS. For LC-MS/MS analysis, the peptides were separated using $90 \mathrm{~min}$ gradient elution at a flow rate of $2.2 \times 10^{-7} \mathrm{l} / \mathrm{min}$ (nitrogen gas temperature, $320^{\circ} \mathrm{C}$; nebulizer pressure, $120 \mathrm{psi}$ ) using a Thermo Scientific $^{\mathrm{TM}}$ EASY-nLC ${ }^{\mathrm{TM}} 1000$ HPLC system (Thermo Fisher Scientific, Inc.), which was directly interfaced with a Thermo Scientific Q Exactive ${ }^{\mathrm{TM}}$ mass spectrometer. Mobile phase A consisted of $0.1 \%$ formic acid and mobile phase B consisted of acetonitrile with $0.1 \%$ formic acid. The Q Exactive ${ }^{\mathrm{TM}}$ mass spectrometer was operated in the data-dependent acquisition mode using Xcalibur 2.2 SP1 software (Thermo Fisher Scientific, Inc.) and there was a single full-scan mass spectrum in the orbitrap (300-2,000 m/z; 70,000 resolution) followed by 20 data-dependent MS/MS scans at $27 \%$ normalized collision energy (HCD). The mass spectrometry proteomics data have been deposited to the ProteomeXchange Consortium via the PRIDE (13) partner repository with the dataset identifier PXD0242499.

Bioinformatics and statistical analysis. A total of 74,811 sequence entries from the human proteome database, 20,350 entries from Swiss-Prot and 544,618 entries from TrEMBL were downloaded from the UniProt Knowledgebase (https://www. uniprot.org/proteomes/UP000005640) on December 20, 2019 and imported into the MaxQuant-associated Andromeda search engine (version 1.6) (14). Following protein identification, the intensity of each identified protein was calculated using peptide signal intensities. A fold-change (FC) $\geq 2$ and $\mathrm{P}<0.01$ were set as the screening benchmark of significantly enhanced or weakened differential expression. Then, the $\operatorname{LogFC}$ value was drawn on the abscissa and the $-\log 10$ (P-value) value was drawn on the ordinate to generate a volcano map. TopGO software (15) (version 2.3.0) was used to conduct the Gene Ontology (GO) (http://geneontology.org/) analysis of the differential proteins from the three levels of biological process, cell component and molecular function. Then, using KOBAS software (16) (version 3.0), the gene ID corresponding to the differential protein was searched using the gene name via BLAST (https://blast.ncbi.nlm.nih.gov/Blast.cgi). Genes 
Table I. Primer sequences for amplification of IFIT3, PARP9, CYB5R1, PTPN1, SLC34A2, DPT, SLPI and GAPDH.

\begin{tabular}{lll}
\hline Genes & \multicolumn{1}{c}{ Forward primers, 5'-3' } & \multicolumn{1}{c}{ Reverse primers, 5'-3' } \\
\hline IFIT3 & GCTGCAAGCAGCCAAATGTT & CTCTGGGACTGGAGCTGACT \\
PARP9 & AGGGAAGAGTGAGCTGGGACAAG & TCTGCCGTCTGCCATTCAATGTG \\
CYB5R1 & TGGCTGTGGGCTCCTACTTGG & GGGCAAAGCGGAACCTCTTGG \\
PTPN1 & TCAAAGTCCGAGAGTCAGGGTCAC & CATCAGCAAGAGGCAGGTATCAGC \\
SLC34A2 & GATGCCGTCGTCTCCAAGTTCAC & TCCTCCAAGTCCTCGCAGCAC \\
DPT & TGGGGCCAGTATGGCGATTA & CTGGTAGCTGAAGCCTTGCC \\
SLPI & GCTGTGGAAGGCTCTGGAAAGTC & CAGTCACTCTGGCACTCAGGTTTC \\
GAPDH & CAGGAGGCATTGCTGATGAT & GAAGGCTGGGGCTCATTT
\end{tabular}

IFIT3, interferon-induced protein with tetratricopeptide repeats 3; PARP9, poly(ADP-ribose) polymerase family member 9; CYB5R1, cytochrome b5 reductase 1; PTPN1, protein tyrosine phosphatase, nonreceptor type 1; SLC34A2, solute carrier family 34 member 2; DPT, dermatopontin; SLPI, secretory leukocyte peptidase inhibitor.

enriched in the corresponding pathways were identified using the Kyoto Encyclopaedia of Genes and Genomes (KEGG) database (http://www.genome.jp/kegg/) and differential proteins were analysed based on KEGG pathway enrichment.

Reverse transcription quantitative PCR (RT-qPCR). In total, $\sim 50 \mathrm{mg}$ of cancer and paracancerous tissue samples were weighed and RNAiso Plus (Takara Biotechnology Co., Ltd.) was added to extract total RNA from the tissue. The concentration and purity of RNA were determined on a NanoDrop ND-1000 Spectrophotometer (Thermo Fisher Scientific, Inc.). Then, the PrimeScript ${ }^{\mathrm{TM}}$ RT kit (Takara Biotechnology Co., Ltd.) was used to perform reverse transcription according to the manufacturer's instructions on an ABI PCR instrument 2720 (Applied Biosystems; Thermo Fisher Scientific, Inc.). A LightCycler ${ }^{\circledR} 480$ II fluorescence quantitative PCR instrument (Roche Diagnostics) and TB Green ${ }^{\circledR}$ Premix Ex Taq ${ }^{\mathrm{TM}}$ II (Takara Biotechnology Co., Ltd.) were used for PCR according to the manufacturers' protocols. The reaction system comprised a mixture of $2.66 \mu \mathrm{lDNA}, 0.6 \mu \mathrm{l}$ upstream primer, $0.67 \mu 1$ downstream primer and $5 \mu$ l fluorescent dye. The amplified genes for the PCR reaction and all required primers synthesized by Sangon Biotech Co., Ltd. were shown in Table I. The experiment was repeated thrice and the $2^{-\Delta \Delta C q}$ method (17) was used to analyse the data.

Western blotting. The main antibodies used included DPT (1:500; cat. no. DF12196; Affinity Biosciences), GAPDH (1:500; cat. no. AP0063; Bioworld Technology, Inc) and horseradish peroxidase-conjugated secondary anti-rabbit IgG (1:1,000; cat. no. BS13278; Bioworld Technology, Inc). Total cancer and paracancerous tissues were lysed with RIPA buffer (Beyotime Institute of Biotechnology), and the total proteins were extracted and determinated using a BCA protein assay kit. Then, 10\% SDS-PAGE was performed to separate proteins, and each lane was loaded with an equal amount of protein $(100 \mu \mathrm{g})$. Subsequently, the protiens were transferred onto the nitrocellulose membrane and blocked with albumin bovine V (Beijing Solarbio Science \& Technology Co., Ltd.) at room temperature for $1.5 \mathrm{~h}$. The strips were incubated with DPT and GAPDH antibodies at $4^{\circ} \mathrm{C}$ overnight. On the second day, the strips were removed, washed thrice with TBST (1x TBS and $0.1 \%$ Tween-20) and incubated with horseradish peroxidase-conjugated secondary antibody at room temperature for $1 \mathrm{~h}$. After incubation, the strips were cleaned thrice and tested and the experiment was repeated thrice for quantitative analysis. The densities and intensities of the strips were quantified using Odyssey ${ }^{\circledR}$ SA (LI-COR; Biosciences).

Immunohistochemical staining. All EC and normal endometrial tissues were fixed in $10 \%$ neutral buffered formalin solution at room temperature for $24 \mathrm{~h}$, then were routinely embedded in paraffin and cut into $4-\mu \mathrm{m}$ thick sections. The sections were quenched with $3 \%$ hydrogen peroxide at room temperature for $10 \mathrm{~min}$, then rinsed in phosphate-buffered saline. Following antigen retrieval in $10 \mathrm{mM}$ citrate buffer for $95^{\circ} \mathrm{C} 5 \mathrm{~min}, 40^{\circ} \mathrm{C} 15 \mathrm{~min}$, the sections were incubated with primary polyclonal antibody DPT (1:100; cat. no. DF12196; Affinity Biosciences) at $4{ }^{\circ} \mathrm{C}$ overnight. The negative control was treated with PBS instead of primary antibody. Then, the secondary antibody, goat anti-rabbit IgG (PV-9001; Beijing Zhongshan Jinqiao Biotechnology Co., Ltd) was applied to the sections at $37^{\circ} \mathrm{C}$ for $20 \mathrm{~min}$. At room temperature, the sections were dyed using diaminobenzidine (DAB) for $60 \mathrm{sec}$, re-stained with haematoxylin for $20 \mathrm{sec}$, and sealed for observation. DPT-positive cells showed yellow-brown granules in the cytoplasm. Images were captured using an Olympus VS120 ${ }^{\circledR}$ digital slice scanning microscope (Olympus Corporation). Then, linear measurement data were acquired using Image-Pro ${ }^{\circledR}$ Plus 6.0 (Media Cybernetics Inc.).

Statistical analysis. All data were presented as the mean \pm the standard error of the mean. The statistical analyses were performed using SPSS 22.0 (IBM Corp.) and GraphPad Prism software version 5.0 (GraphPad Software, Inc.). A paired t-test was used for comparisons between cancer tissues and paracancerous tissues from the same patient, and an independent sample t-test was used for comparisons between normal endometrial and EC cases. The associations between the expression of DPT and clinicopathological features were evaluated using $\chi^{2}$ and Fisher's exact tests. $\mathrm{P}<0.05$ was considered to indicate a statistically significant difference. 


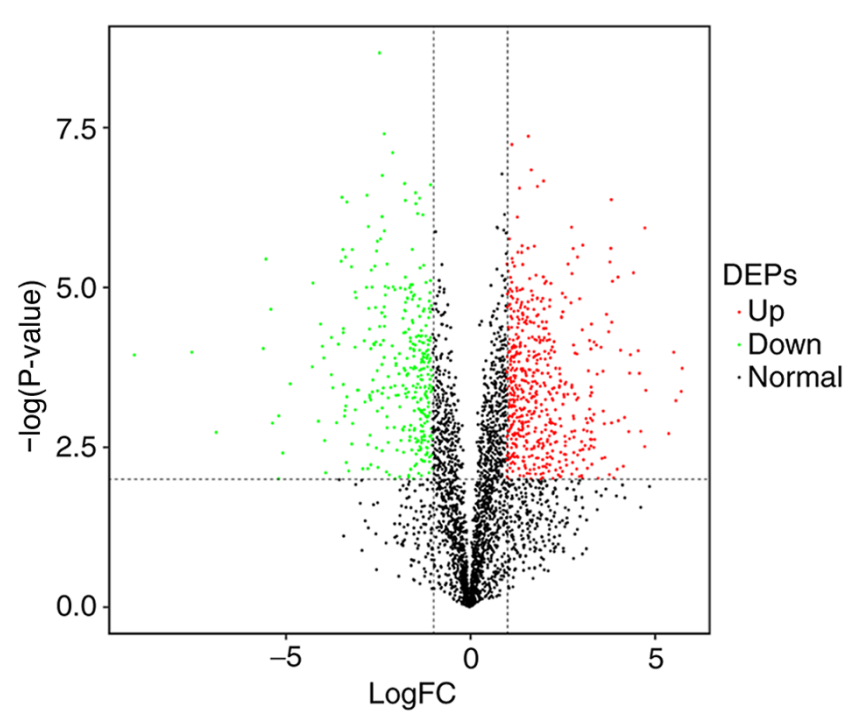

Figure 1. Volcanic map of DEPs based on FC $\geq 2$ and $\mathrm{P}<0.01$. Red dots represent 579 up-regulated proteins and green dots represent 346 down-regulated proteins. DEPs, differentially expressed proteins; up, up-regulated; down, down-regulated; FC, fold-change.

\section{Results}

Proteomic analysis of EC. After the cancer and paracancerous tissue samples were hydrolysed and pre-treated, the total differential proteins were determined by LC-MS/MS and analysed by bioinformatics and statistical analysis. A majority of the identified peptides were distributed in 7-25 amino acids, which was consistent with the general laws of enzymatic hydrolysis and HCD fragmentation. Through quantitative screening using MaxQuant a total of 3,401 proteome samples were retrieved from the two groups, among which the quantifiable number of proteins were 3,245 (Table SI). Student's t-tests were conducted after the two groups were classified and screened. In total, 579 proteins were significantly up-regulated and 346 proteins were significantly down-regulated (Tables II and SII). Eventually, 925 differential proteins were screened as differentially expressed proteins (DEPs) as shown in a volcano map (Fig. 1). The red dots represent 579 significantly up-regulated proteins, whereas the green dots represent 346 significantly down-regulated proteins.

To analyse the molecular functions of the identified proteins, DEPs were clustered into GO categories, including biological process, cellular components and molecular functions. The up-regulated DEPs were primarily distributed in the 'membrane' and 'extracellular exosome', while the down-regulated DEPs were mainly located in 'extracellular exosome' and 'extracellular matrix', as shown in Fig. 2A and B, respectively. The functional enrichment of DEPs was also analysed using the KEGG database. Up-regulated DEPs are mainly involved in 'protein processing in the endoplasmic reticulum' and 'phagosome', while down-regulated DEPs played a crucial role in 'ribosome' and 'focal adhesion', as seen in Fig. 2C and D, respectively. This finding is consistent with the data described in the subsequent paragraphs. As aforementioned, DPT is an extracellular matrix protein, which principally takes a significant role in focal adhesion,
Table II. Total up-regulated or down-regulated proteins.

\begin{tabular}{llc}
\hline $\begin{array}{l}\text { Compared } \\
\text { groups }\end{array}$ & $\begin{array}{c}\text { Regulation } \\
\text { type }\end{array}$ & $\begin{array}{c}\text { Fold-changes } \\
\geq 2 \text { and } \mathrm{P}<0.01\end{array}$ \\
\hline EC/AP & Up-regulated & 579 \\
& Down-regulated & 346 \\
\hline
\end{tabular}

EC, endometrial cancer; AP, paracancerous tissue.

and its down-regulation can accelerate tumour invasion and progression (18).

Identification of diagnostic and prognostic biomarkers. The seven potential predictors of EC were chosen according to the difference ratio of proteins $(>5 ; \mathrm{P}<0.01)$ protein function and literatures and classified into two groups: Paracancerous (AP) or EC. The up-regulated proteins included interferon-induced protein with tetratricopeptide repeats 3 (IFIT3), poly(ADP-ribose) polymerase family member 9 (PARP9), solute carrier family 34 member 2 (SLC34A2), cytochrome b5 reductase 1 (CYB5R1) and protein tyrosine phosphatase non-receptor type 1 (PTPN1), while down-regulated proteins contained DPT and secretory leukocyte peptidase inhibitor (SLPI) (Table III). The RT-qPCR results of differential proteins are presented in Fig. 3. IFIT3, PARP9, CYB5R1, PTPN1 and SLPI expressions were not conspicuously up-regulated or down-regulated, whereas SLC34A2 and DPT expressions in EC were significantly decreased $(\mathrm{P}<0.05$ and $\mathrm{P}<0.001$, respectively; Fig. $3 \mathrm{E}$ and $\mathrm{F}$ ). Western blotting results showed that the DPT in endometrial carcinoma was significantly lower compared with that in paracancerous tissues $(\mathrm{P}<0.001$; Fig. 4). In summary, the DPT results were consistent in LC-MS/MS, RT-qPCR and western blotting DPT was shown to be significantly down-regulated in EC. These findings indicated that the expression of DPT was markedly decreased in EC, which might be closely associated with the pathogenesis of EC.

Association between DPT and clinicopathological characteristics. A total of $75 \mathrm{EC}$ tissue wax blocks included 54 patients with stage I-II EC and 21 patients with stage III-IV. There were 49 patients with high and medium differentiation and 26 patients with low differentiation. The aforementioned results indicated that DPT might play a role in inhibiting progression in EC and that its reduction promotes cancer migration and progression. The immunohistochemical staining results of the wax blocks of 30 normal endometrial (normal group) and 75 EC tissues (EC group) are provided in Fig. 5. In 30 cases of normal endometrial tissues, 25 cases were positive and the rest were absent. Among the 75 EC tissues, 58 cases showed low expression and only 17 cases showed high-expression. Compared with normal endometrium, DPT was markedly down-regulated in EC tissues. Afterwards, the $\chi^{2}$ and Fisher's exact tests were applied to evaluate the association between DPT and clinicopathological features (Table IV). Staging, differentiation, depth of myometrial invasion and lymph node metastasis were found to be associated with DPT expression. 
A

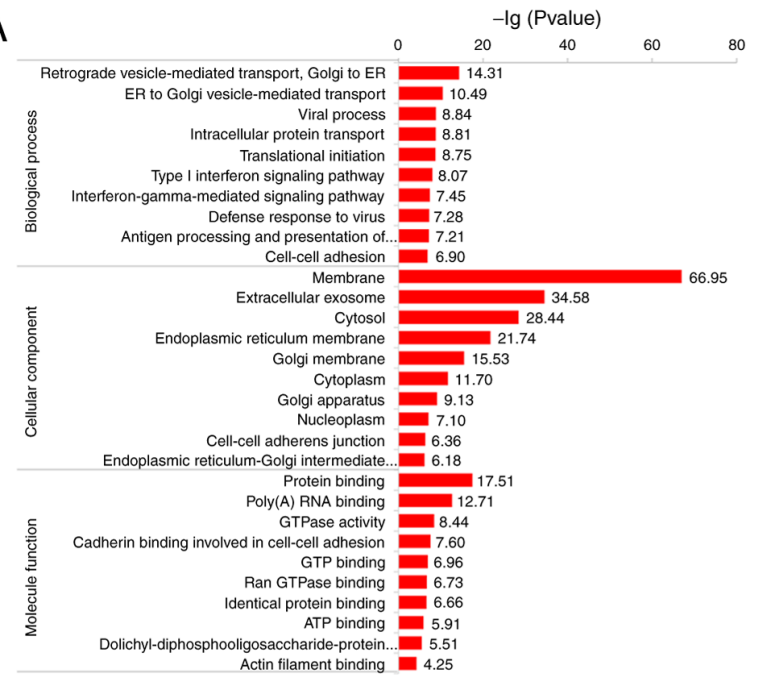

C

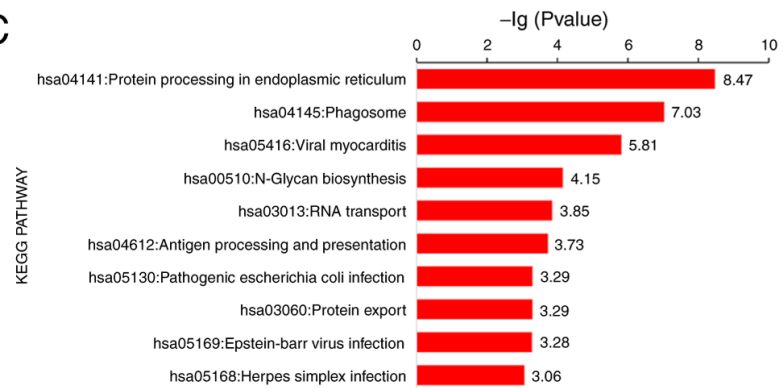

B
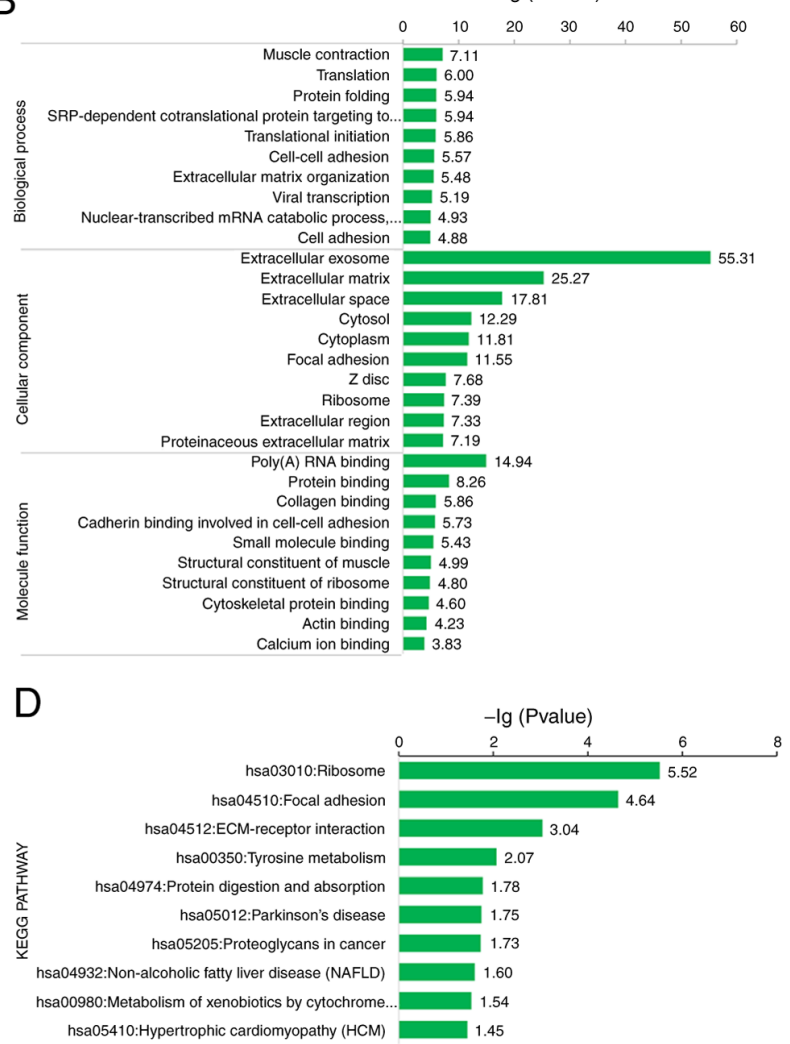

Figure 2. Bioinformatics analysis of differential proteins. (A and B) Gene Ontology enrichment analysis of differential proteins was analysed at three levels: Biological process, cell composition and molecular function. (C and D) KEGG pathway enrichment analysis of differentially expressed proteins. KEGG, Kyoto Encyclopaedia of Genes and Genomes.
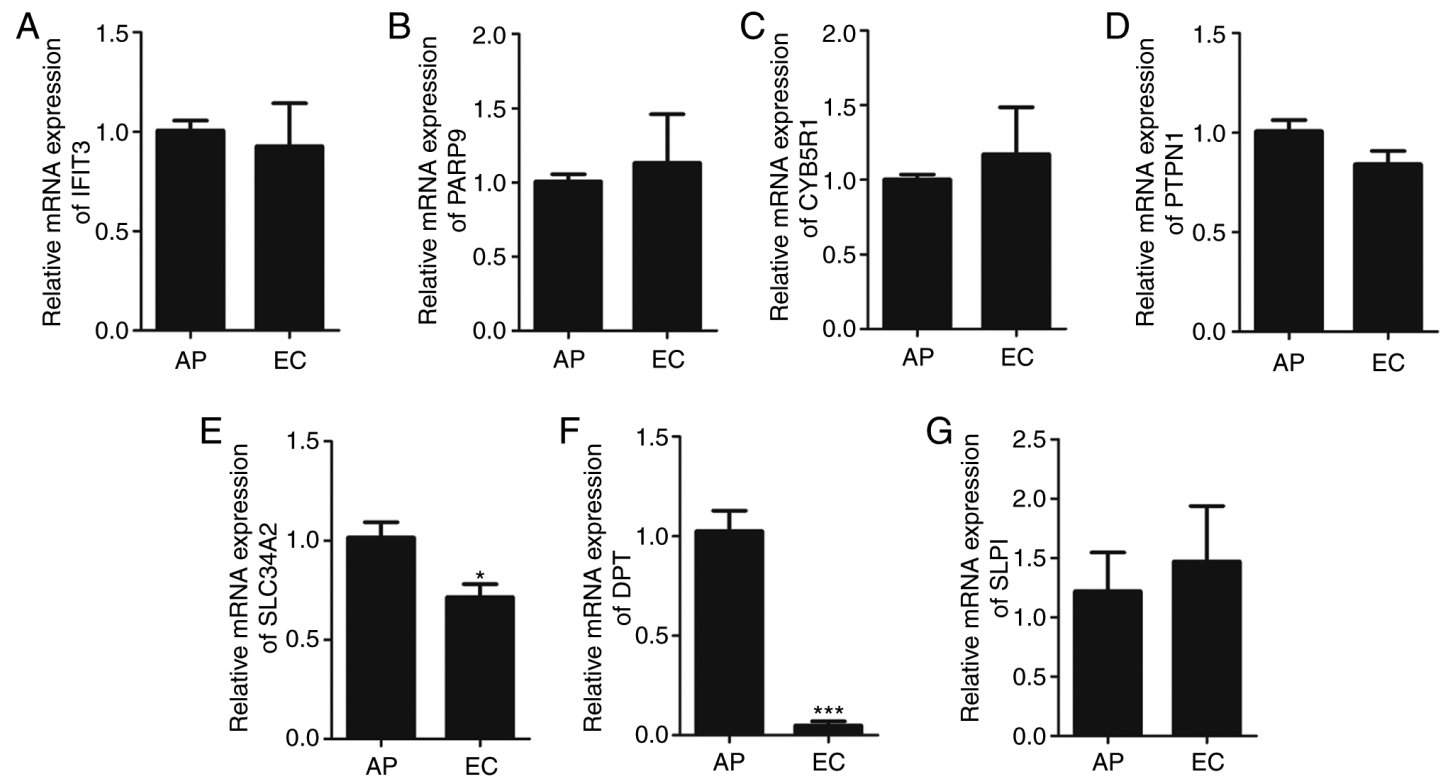

Figure 3. mRNA expression of differential proteins in EC and AP tissues. (A) IFIT3. (B) PARP9. (C) CYB5R1. (D) PTPN1. (E) SLC34A2. (F) DPT. (G) SLPI SLC34A2 and DPT expressions were significantly down-regulated in cancer tissues. ${ }^{*} \mathrm{P}<0.05$ and ${ }^{* * * *} \mathrm{P}<0.001$ compared with the AP group. EC, endometrial cancer; AP, paracancerous; IFIT3, interferon-induced protein with tetratricopeptide repeats 3; PARP9, poly(ADP-ribose) polymerase family member 9; CYB5R1, cytochrome b5 reductase 1; PTPN1, protein tyrosine phosphatase nonreceptor type 1; SLC34A2, solute carrier family 34 member 2; DPT, dermatopontin; SLPI, secretory leukocyte peptidase inhibitor.

As shown in Table IV, the positive expression of DPT was markedly decreased in tissues with late stage, poor differentiation, myometrial invasion depth $\geq 1 / 2$ and lymph node metastasis $(\mathrm{P}<0.05)$. The above results demonstrated that the positive expression of DPT decreased with increasing tumour malignancy. 

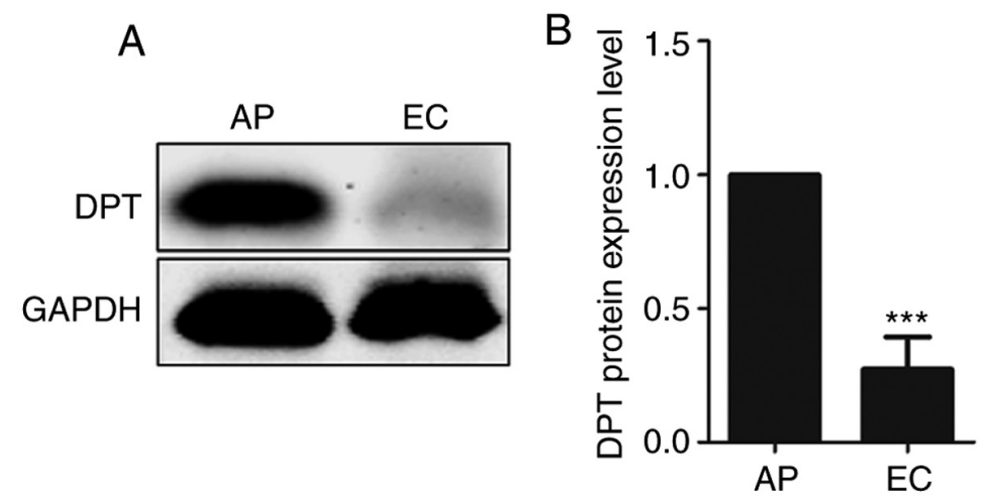

Figure 4. DPT protein expression in EC and AP tissues. (A) Representative western blotting results of DPT. (B) Quantified DPT expression. ${ }^{* * *} \mathrm{P}<0.001$ compared with the AP group. DPT, dermatopontin; EC, endometrial cancer; AP, paracancerous.
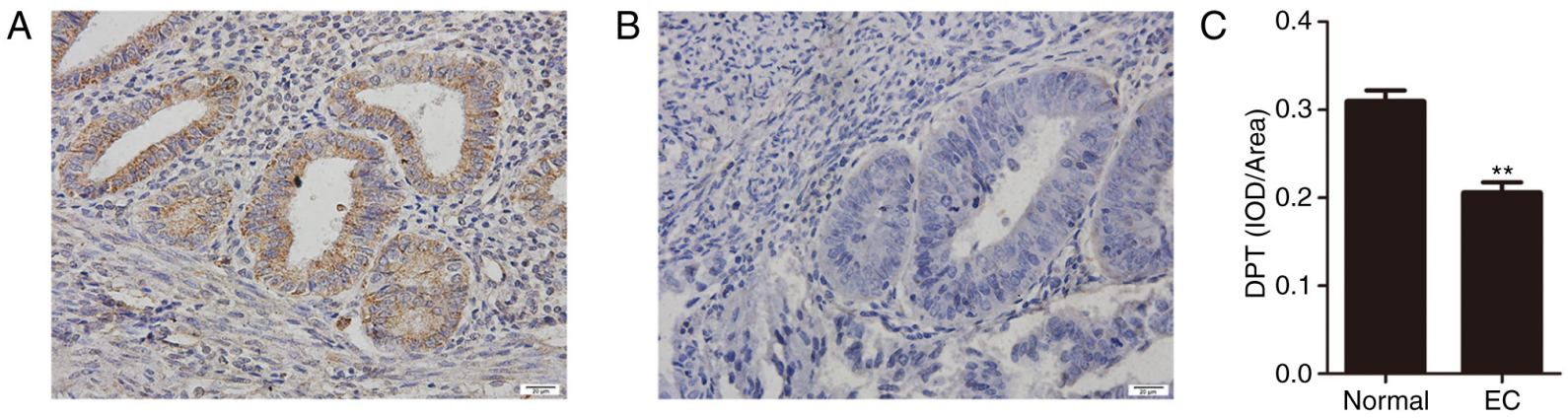

Figure 5. Expression of DPT in normal endometrial tissues and EC tissues. (A and B) Representative images of DPT immunohistochemical staining in normal endometrial tissues $(n=30)$ and EC tissues $(n=75)$ (original magnification, $x 400)$. (A) Normal endometrial tissues; (B) EC tissues. (C) Quantified DPT immunohistochemical staining. Values are expressed as the mean + the standard error of the mean. ${ }^{* *} \mathrm{P}<0.05$ compared with the normal endometrial tissues. DPT, dermatopontin; EC, endometrial cancer; IOD, integrated optical density.

\section{Discussion}

The proteome of EC was detected based on LC-MS/MS and DEPs were analysed using bioinformatics. In total, seven DEPs were identified, including IFIT3, PARP9, SLC34A2, CYB5R1, PTPN1, DPT and SLPI. RT-qPCR and western blotting showed that DPT expression was significantly decreased in EC. DPT expression in 75 cases of endometrial carcinoma and 30 cases of normal non-cancerous endometrium was detected using immunohistochemical staining, and the association between the expression and clinicopathological factors was analysed. Compared with normal endometrium, DPT expression in cancer tissues was decreased, and DPT was significantly associated with differentiation, FIGO stage, muscle infiltration depth and lymph node metastasis.

The extracellular matrix is a macromolecular dynamic reticular structure composed of collagen, proteoglycans and other glycoproteins (19). The migration of cancer cells depends on the biochemical characteristics of the ECM, while integrin $\alpha 3 \beta 1$ can promote cancer cell migration and invasion (20). After destroying the basement membrane, the tumour cells move along the track of collagen fibres, invade the vascular endothelial basement membrane and finally reach distant organs through the circulation (21). Tumour cells can also migrate through the network around the extracellular matrix. Even in primary tumours, the extracellular matrix is constantly altered under the influence of the tumour microenvironment, thus promoting tumour migration and disease progression $(21,22)$.

DPT is an extracellular matrix protein that plays an important role in matrix remodelling and metastasis of cancer tissues. DPT is expressed at a low level in oral squamous cell carcinoma, hepatocellular carcinoma and thyroid papillary carcinoma and can promote the proliferation of prostate cancer cells $(18,23)$. DPT not only increases the biological activity of transforming growth factor $\beta$ but also combines with integrin $\alpha 3 \beta 1$ to promote the germination of tumour neovascularization and ducts by enhancing the movement ability of tumour cells and inducing the formation of endothelial cell adhesion $(9,11)$. Fu et al (24) found that low DPT expression in hepatocellular carcinoma is mainly mediated by DNA methylation and also demonstrated that DPT enhances the stability of focal adhesion through $\alpha 3 \beta 1$ integrin-Rho GTPase signalling, thus inhibiting the metastasis of hepatocellular carcinoma. In thyroid carcinoma, ectopic expression of DPT hinders thyroid cancer cell proliferation (25). DPT also down-regulates the expression of c-Myc and regulates the expression of cell cycle-dependent kinases (CDK4 and CDK6) and cyclin-dependent kinase inhibitors (P21) through the MEK-ERK-MYC signalling pathway (25).

Despite a low expression pattern of DPT in numerous types of cancer, no related studies have been reported in endometrial carcinoma, to the best of our knowledge. In the present study, DPT expression in endometrial carcinoma was studied and its association with clinicopathological factors was 
Table III. Seven differential proteins enriched in bioinformatics analysis.

\begin{tabular}{|c|c|c|c|c|}
\hline $\begin{array}{l}\text { Registration number } \\
\text { in UniProt database }\end{array}$ & Name of differential proteins & Gene & Regulation & P-value \\
\hline O14879 & Interferon-induced protein with tetratricopeptide repeats 3 & IFIT3 & Up & 0.00474 \\
\hline Q8IXQ6 & Poly(ADP-ribose) polymerase family member 9 & PARP9 & Up & 0.00178 \\
\hline Q9UHQ9 & Cytochrome b5 reductase 1 & CYB5R1 & $\mathrm{Up}$ & 0.00460 \\
\hline P18031 & Protein tyrosine phosphatase, nonreceptor type 1 & PTPN1 & Up & 0.00239 \\
\hline O95436 & Solute carrier family 34 member 2 & SLC34A2 & Up & 0.00630 \\
\hline Q07507 & Dermatopontin & DPT & Down & 0.00001 \\
\hline P03973 & Secretory leukocyte peptidase inhibitor & SLPI & Down & 0.00390 \\
\hline
\end{tabular}

Table IV. Association between DPT expression and clinicopathological features of patients with endometrial cancer.

\begin{tabular}{|c|c|c|c|c|c|}
\hline \multirow[b]{2}{*}{ Clinical features } & \multirow[b]{2}{*}{ Cases, $\mathrm{n}$} & \multicolumn{2}{|c|}{ DPT } & \multirow[b]{2}{*}{$\chi^{2}$} & \multirow[b]{2}{*}{ P-value } \\
\hline & & High & Low & & \\
\hline FIGO stage & & & & - & 0.029 \\
\hline I-II & 54 & 16 & 38 & & \\
\hline III-IV & 21 & 1 & 20 & & \\
\hline Differentiation & & & & 5.091 & 0.024 \\
\hline High and medium & 49 & 15 & 34 & & \\
\hline Poor & 26 & 2 & 24 & & \\
\hline Depth of muscular invasion & & & & 6.799 & 0.009 \\
\hline$<1 / 2$ & 41 & 14 & 27 & & \\
\hline$\geq 1 / 2$ & 34 & 3 & 31 & & \\
\hline Lymph node metastasis & & & & - & 0.031 \\
\hline No & 55 & 16 & 39 & & \\
\hline Yes & 20 & 1 & 19 & & \\
\hline
\end{tabular}

DPT, dermatopontin; FIGO, International Federation of Gynaecology and Obstetrics.

analysed. LC-MS/MS, RT-qPCR and western blotting results all confirmed a low level of DPT expression in endometrial carcinoma. The expression of DPT was decreased in poorly differentiated tumours, more advanced clinicopathological stages, deeper myometrial invasion and in cases of lymph node metastasis. Integrins are a type of cell adhesion molecule that depend on $\mathrm{C}^{\mathrm{a} 2+}$ or $\mathrm{Mg}^{2+}$ to mediate recognition and adhesion between cells and the extracellular matrix and DPT mediates cell adhesion by binding to integrin $\alpha 3 \beta 1$, thus inhibiting the proliferation and migration of tumour cells (24). Therefore, it was hypothesised that DPT might be involved in the pathogenesis of endometrial carcinoma and that its exact molecular mechanism needs to be further studied.

However, the present study only contained the most common pathological types of endometrial carcinoma and not rare pathological types, such as serous carcinoma, mucinous carcinoma, clear cell carcinoma and carcinosarcoma. Due to the limitation of the sample size and single pathological type, further research should be conducted to reveal more prevalent phenomena. Notably, the current study confirmed for the first time that decreased DPT levels were associated with EC, so identifying the expression of
DPT in EC might contribute to identifying novel biomarkers and providing future prognostic guidance. In-depth exploration of DPT may reveal the molecular mechanism of EC and provide new ideas for targeted therapy to increase the success of treatment and survival rate of patients with advanced EC.

\section{Acknowledgements}

Not applicable.

\section{Funding}

This work was supported by The Jiangsu Provincial Commission of Health and Family Planning (grant no. H2017079) and The Jiangsu Science and Technology Planning Project (grant no. BE2019636).

\section{Availability of data and materials}

The datasets used and analysed during the current study are available from the corresponding author on reasonable 
request. The LC-MS/MS datasets generated during the current study are available in the PRIDE repository (http://www.ebi. ac.uk/pride). The datasets generated and/or analysed during the current study are available in the UniProt knowledgebase (https:/www.uniprot.org/proteomes/UP000005640).

\section{Authors' contributions}

$\mathrm{XZ}, \mathrm{HH}$ and $\mathrm{BZ}$ participated in the conception and design of this study. $\mathrm{HH}, \mathrm{ZH}, \mathrm{LL}$ and $\mathrm{CW}$ carried out the experiments. $\mathrm{HH}$ and $\mathrm{ZH}$ collected the experimental data. $\mathrm{HH}, \mathrm{ZH}$ and $\mathrm{ZY}$ analysed and interpreted the data. HH, ZY, XZ and BZ took charge of writing the article and revising it. $\mathrm{HH}, \mathrm{ZH}$ and $\mathrm{XZ}$ confirm the authenticity of all raw data. All the authors read and approved the final manuscript.

\section{Ethics approval and consent to participate}

This study was approved by The Ethics Committee of Xuzhou Central Hospital. The patients included in the study provided written consent.

\section{Patient consent for publication}

Not applicable.

\section{Competing interests}

The authors declare that they have no competing interests.

\section{References}

1. Njoku K, Chiasserini D, Whetton AD and Crosbie EJ: Proteomic biomarkers for the detection of endometrial cancer. Cancers (Basel) 11: 1572, 2019.

2. Miller KD, Nogueira L, Mariotto AB, Rowland JH, Yabroff KR, Alfano CM, Jemal A, Kramer JL and Siegel RL: Cancer treatment and survivorship statistics, 2019. CA Cancer J Clin 69: 363-385, 2019.

3. Martinez-Garcia E, Lopez-Gil C, Campoy I, Vallve J, Coll E, Cabrera S, Ramon Y Cajal S, Matias-Guiu X, Van Oostrum J, Reventos J, et al: Advances in endometrial cancer protein biomarkers for use in the clinic. Expert Rev Proteomics 15: 81-99, 2018.

4. Maxwell GL, Hood BL, Day R, Chandran U, Kirchner D, Kolli VS, Bateman NW, Allard J, Miller C, Sun M, et al: Proteomic analysis of stage I endometrial cancer tissue: Identification of proteins associated with oxidative processes and inflammation. Gynecol Oncol 121: 586-594, 2011.

5. Ueda SM, Kapp DS, Cheung MK, Shin JY, Osann K, Husain A, Teng NN, Berek JS and Chan JK: Trends in demographic and clinical characteristics in women diagnosed with corpus cancer and their potential impact on the increasing number of deaths. Am J Obstet Gynecol 198: 218.e1-218.e6, 2008.

6. Giorgianni F, Koirala D and Beranova-Giorgianni S: Proteomics of the human pituitary tissue: Bioanalytical methods and applications. Bioanalysis 6: 1989-2003, 2014.

7. Peng L, Cantor DI, Huang C, Wang K, Baker MS and Nice EC: Tissue and plasma proteomics for early stage cancer detection. Mol Omics 14: 405-423, 2018.
8. Neame PJ, Choi HU and Rosenberg LC: The isolation and primary structure of a $22-\mathrm{kDa}$ extracellular matrix protein from bovine skin. J Biol Chem 264: 5474-5479, 1989.

9. Okamoto O, Fujiwara S, Abe M and Sato Y: Dermatopontin interacts with transforming growth factor beta and enhances its biological activity. Biochem J 337: 537-541, 1999.

10. Okamoto O, Hozumi K, Katagiri F, Takahashi N, Sumiyoshi H, Matsuo N, Yoshioka H, Nomizu M and Fujiwara S: Dermatopontin promotes epidermal keratinocyte adhesion via $\alpha 3 \beta 1$ integrin and a proteoglycan receptor. Biochemistry 49: 147-155, 2010.

11. Krishnaswamy VR, Balaguru UM, Chatterjee S and Korrapati PS: Dermatopontin augments angiogenesis and modulates the expression of transforming growth factor beta 1 and integrin alpha 3 beta 1 in endothelial cells. Eur J Cell Biol 96: 266-275, 2017.

12. Mutch D: I231 FIGO Staging of Endometrial Cancer 2009. Int J Gynaecol Obstet 107: S58-S58, 2009.

13. Perez-Riverol Y, Csordas A, Bai J, Bernal-Llinares M, Hewapathirana S, Kundu DJ, Inuganti A, Griss J, Mayer G, Eisenacher M, et al: The PRIDE database and related tools and resources in 2019: Improving support for quantification data. Nucleic Acids Res 47: D442-D450, 2019.

14. Cox J, Neuhauser N, Michalski A, Scheltema RA, Olsen JV and Mann M: Andromeda: A peptide search engine integrated into the MaxQuant environment. J Proteome Res 10: 1794-1805, 2011.

15. Alexa A, Rahnenführer J and Lengauer T: Improved scoring of functional groups from gene expression data by decorrelating GO graph structure. Bioinformatics 22: 1600-1607, 2006.

16. Xie C, Mao X, Huang J, Ding Y, Wu J, Dong S, Kong L, Gao G, Li CY and Wei L: KOBAS 2.0: A web server for annotation and identification of enriched pathways and diseases. Nucleic Acids Res 39 (Supp1 2): W316-W322, 2011.

17. Livak KJ and Schmittgen TD: Analysis of relative gene expression data using real-time quantitative PCR and the 2(- $\Delta \Delta \mathrm{C}(\mathrm{T}))$ method. Methods 25: 402-408, 2001.

18. Takeuchi T, Suzuki M, Kumagai J, Kamijo T, Sakai M and Kitamura T: Extracellular matrix dermatopontin modulates prostate cell growth in vivo. J Endocrinol 190: 351-361, 2006.

19. Theocharis AD, Skandalis SS, Gialeli C and Karamanos NK: Extracellular matrix structure. Adv Drug Deliv Rev 97: 4-27, 2016.

20. Cavaco ACM, Rezaei M, Caliandro MF, Lima AM, Stehling M, Dhayat SA, Haier J, Brakebusch C and Eble JA: The interaction between laminin-332 and $\alpha 3 \beta 1$ integrin determines differentiation and maintenance of CAFs, and supports invasion of pancreatic duct adenocarcinoma cells. Cancers (Basel) 11: 14, 2018.

21. Eble JA and Niland S: The extracellular matrix in tumor progression and metastasis. Clin Exp Metastasis 36: 171-198, 2019.

22. Kalluri R: The biology and function of fibroblasts in cancer. Nat Rev Cancer 16: 582-598, 2016.

23. Yamatoji M, Kasamatsu A, Kouzu Y, Koike H, Sakamoto Y, Ogawara K, Shiiba M, Tanzawa H and Uzawa K: Dermatopontin: A potential predictor for metastasis of human oral cancer. Int $\mathrm{J}$ Cancer 130: 2903-2911, 2012.

24. Fu Y, Feng M-X, Yu J, Ma MZ, Liu XJ, Li J, Yang XM, Wang YH, Zhang YL, Ao JP, et al: DNA methylation-mediated silencing of matricellular protein dermatopontin promotes hepatocellular carcinoma metastasis by $\alpha 3 \beta 1$ integrin-Rho GTPase signaling. Oncotarget 5: 6701-6715, 2014.

25. Guo Y, Li H, Guan H, Ke W, Liang W, Xiao H and Li Y: Dermatopontin inhibits papillary thyroid cancer cell proliferation through MYC repression. Mol Cell Endocrinol 480: 122-132, 2019.

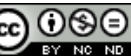

This work is licensed under a Creative Commons Attribution-NonCommercial-NoDerivatives 4.0 International (CC BY-NC-ND 4.0) License. 